\title{
Signalling pathways implicated in the pathogenesis of rheumatic heart disease (Review)
}

\author{
SHENGLIN XIAN ${ }^{1,2}$ and ZHIYU ZENG ${ }^{1,2}$ \\ ${ }^{1}$ Department of Cardiology, The First Affiliated Hospital of Guangxi Medical University; ${ }^{2}$ Guangxi Key Laboratory of \\ Precision Medicine in Cardio-cerebrovascular Disease Control and Prevention and Guangxi Clinical Research Center \\ for Cardio-cerebrovascular Diseases, Guangxi Medical University, Nanning, Guangxi 530021, P.R. China
}

Received July 27, 2020; Accepted October 28, 2020

DOI: $10.3892 /$ etm.2020.9508

\begin{abstract}
Rheumatic heart disease (RHD) is frequently encountered in underdeveloped areas and primarily affects patients under the age of 40 years old. The pathogenesis of RHD has yet to be fully elucidated and surgical treatment remains the only option, which is expensive and technically demanding for patients in less developed areas. Signalling pathways are crucial for the occurrence and development of several diseases, and researchers worldwide have made progress in elucidating the signalling pathways associated with the pathogenesis of RHD. The aim of the present review was to discuss 6 signalling pathways implicated in the pathogenesis of RHD, summarize the methods and progress of these studies and propose future research directions. Important information on the pathogenesis of RHD according to the current progress of signalling pathway studies was also summarized, in the hope that this review may serve as a reference for future research on the signalling pathways involved in the pathogenesis of RHD.
\end{abstract}

Correspondence to: Professor Zhiyu Zeng, Department of Cardiology, The First Affiliated Hospital of Guangxi Medical University, 6 Shuangyong Road, Nanning, Guangxi 530021, P.R. China

E-mail: doctorzyz@126.com

Abbreviations: RHD, rheumatic heart disease; RhoA/ROCK, RhoA/Rho-associated protein kinase; ECM, extracellular matrix; TN-C, tenascin-C; IFN, interferon; TNF, tumour necrosis factor; MAPK, mitogen-activated protein kinase; AF, atrial fibrillation; SR, sinus rhythm; bFGF, basic fibroblast growth factor; TGF, transforming growth factor; TRAF, tumour necrosis factor receptor-associated factor; AKT/S6K, protein kinase B/S6 kinase; FAK, focal adhesion kinase; $\alpha$-SMA, $\alpha$-smooth muscle actin; EMT, endothelial-to-mesenchymal transition; STAT3, signal transducer and activator of transcription 3; S1PR1, sphingosine 1-phosphate receptor 1; p-STAT3, phosphorylated STAT3; Th17, T helper 17 cells; IL, interleukin

Key words: rheumatic heart disease, signalling pathway, pathogenesis

\section{Contents}

1. Introduction

2. Selection and search criteria

3. Discussion and conclusions

\section{Introduction}

Rheumatic heart disease (RHD) poses a major threat to the health of primarily patients under the age of 40 years old (1). As many as 250,000 people succumb to RHD annually (2). Although valve replacement surgery is currently the most effective treatment, this surgery is expensive and requires high technical skills and specialized equipment. Therefore, RHD represents a major health concern in less developed regions. In order to effectively treat RHD, it is important to fully elucidate its pathogenic process; however, the exact pathogenesis of RHD remains elusive (3). Signalling pathways are key factors in the occurrence and development of several diseases, and research on signalling pathways is an important approach to elucidating the pathogenesis of disease. A number of drugs exert their pharmacological effects by acting on signalling pathways (4-6). To date, there are no effective drugs for the prevention or treatment of RHD. Therefore, it is crucial to study the signalling pathways involved in RHD pathogenesis, as drug intervention in RHD-related pathways for prevention or treatment would markedly benefit RHD patients in underdeveloped areas. Previous studies have investigated the signalling pathways associated with the pathogenesis of RHD. Therefore, the aim of the present review was to discuss the research progress on 6 major signalling pathways implicated in the pathogenesis of RHD, to summarize the available results in order to further elucidate the pathogenesis of RHD, and to serve as a reference for researchers worldwide investigating the pathogenesis of RHD.

\section{Selection and search criteria}

English literature databases were searched using the following terms: 'RHD' (all fields) and 'pathway or signal' (all fields). The following criteria were considered for the review of studies: i) The research content was signalling pathways 
associated with the pathogenesis of RHD; and ii) the research made significant progress, such as the discovery of a certain RHD-related signalling pathway, or examined the mechanism of the RHD-related signalling pathway. The number of studies on the signalling pathways associated with the pathogenesis of RHD worldwide is limited. A total of 6 important signalling pathways that are implicated in the pathogenesis of RHD and on which significant research progress was made were identified.

RhoA/Rho-associated protein kinase (RhoA/ROCK) signalling pathway and RHD. In 2008, Jiang et al (7) investigated the association between extracellular matrix (ECM) and RHD. First, it was observed that the concentration of tenascin-C (TN-C), which is the key component of the ECM in patients with RHD, was significantly higher compared with that in controls. The transcription of TN-C is closely associated with the RhoA/ROCK signalling pathway. Therefore, it was ultimately discovered that interferon (IFN) $-\gamma$ and tumour necrosis factor (TNF)- $\alpha$ were involved in the pathogenesis of RHD via the RhoA/ROCK signalling pathway, which regulated the expression of TN-C and the ECM remodelling process (8). That study identified two important factors associated with RHD: The RhoA/ROCK signalling pathway and the ECM. The RhoA/ROCK signalling pathway regulates an important component of the ECM, as mentioned earlier, but it is also involved in TNF- $\alpha$-mediated endothelial apoptosis (9) and the development of the sinoatrial node (10). Previous studies demonstrated that ECM remodelling in the heart was implicated in cardiac fibrosis (11), and the ECM is also a therapeutic target for myocardial repair (12). Whether the RhoA/ROCK signalling pathway and ECM cooperate with endothelial apoptosis, cardiac fibrosis and myocardial repair in the pathogenesis of RHD is unclear, and it may be investigated to further elucidate the pathogenesis of RHD in the future. A previous study (7) also highlighted two intervention points: Neutralization of IFN- $\gamma$ and TNF- $\alpha$ with antibodies attenuated TN-C transcription in patients with RHD, as IFN- $\gamma$ and TNF- $\alpha$ induce TN-C transcription via the RhoA/ROCK signalling pathway. A number of studies demonstrated that IFN- $\gamma$ and TNF- $\alpha$ were closely associated with the pathogenesis of RHD (13-16). Therefore, IFN- $\gamma$ and TNF- $\alpha$ may be potential targets for therapeutic intervention in RHD. However, a previous study (7) only revealed that neutralization of IFN- $\gamma$ and TNF- $\alpha$ suppressed the transcription of TN-C, but it did not investigate the effects of the lower transcription of TN-C on the occurrence and development of RHD. IFN- $\gamma$ and TNF- $\alpha$ as potential targets for intervention require further investigation in future studies.

Mitogen-activated protein kinase (MAPK) signalling pathway and RHD. In 2013, Li et al (17) divided patients with RHD into atrial fibrillation (AF) and sinus rhythm (SR) groups according to their heart rhythm. When comparing the expression levels of basic fibroblast growth factor (bFGF)-related factors and MAPK signalling pathway-related factors between these two groups, they were found to be higher in the AF group. It was concluded that bFGF may be involved in the process of atrial fibrosis, and the MAPK signalling pathway may be the molecular basis of this process. The focus of that study was atrial fibrosis in patients with RHD, and the association among bFGF, the MAPK signalling pathway and RHD was initially examined.

A 2014 study also examined the role of the MAPK pathway in the process of atrial fibrosis in patients with RHD. Zhang et al (18) also compared the SR and AF groups of RHD patients. The sample size was larger, and the focus was on transforming growth factor (TGF)- $\beta 1$ and tumour necrosis factor receptor-associated factor (TRAF) 6 in addition to MAPK. It was demonstrated that the MAPK/TGF- $\beta 1 / T R A F 6$ signalling pathway was involved in atrial fibrosis in patients with chronic AF, indicating that TRAF6 may be a new intervention target for the treatment of atrial fibrosis. The study also examined the role of the MAPK pathway in atrial fibrosis due to RHD.

In 2020, Zhao et al (19) also investigated the association between the MAPK pathway and RHD. The pathological process investigated in that study was RHD-induced valve fibrosis. Blood samples and valve specimens from patients with RHD were compared to age- and sex-matched RHD-negative patients. The research focused on $\mathrm{CD}^{+} \mathrm{T}$ cells, the MAPK signalling pathway and TGF- $\beta 1$. The findings confirmed that the MAPK signalling pathway and TGF- $\beta 1$ participated in the process of RHD-induced valve fibrosis, and concluded that $\mathrm{CD}^{+} \mathrm{T}$ cells also played an important role in the fibrotic process.

Therefore, the MAPK signalling pathway plays an important role in the process of RHD-induced fibrosis. MAPK is a cellular serine/threonine protein kinase that plays a key role in biological reactions involved in cell proliferation, differentiation, transformation and apoptosis (20). All three aforementioned studies examined the close association between the MAPK signalling pathway and the process of fibrosis of cardiac tissue caused by RHD. The three most important factors in the fibrotic process of RHD involved with MAPK were bFGF, TGF- $\beta 1$ and $\mathrm{CD}^{+} \mathrm{T}$ cells. bFGF promoted the growth of fibroblasts and was closely associated with the pathological process of atrial fibrosis caused by RHD. These studies also demonstrated that the concentrations of bFGF and MAPK were increased in the AF group, but no specific mechanism was further examined, and no suitable intervention target was identified. TGF- $\beta 1$ is known to play an important role in the pathogenesis of RHD, which is discussed below. $\mathrm{CD}^{+} \mathrm{T}$ cells are important immune cells in the human body. It is widely hypothesised that immunity plays a key role in the pathogenesis of RHD. However, the types of immune cells that are involved in this process are not known, although a role for $\mathrm{CD}^{+}{ }^{+} \mathrm{T}$ cells has been suggested. The studies (17-19) aforementioned only reported that the numbers of $\mathrm{CD}^{+} \mathrm{T}$ cells were significantly increased in the peripheral blood and valve tissues of patients with RHD, but did not further examine their mechanism of action. The mechanism of action of $\mathrm{CD}^{+}$ T cells in RHD was later examined, which is discussed below.

Protein kinase B(AKT)/S6 kinase (S6K) signalling pathway and RHD. In 2013, Zhang et al (21) found that focal adhesion kinase (FAK) regulated the AKT/S6K pathway and participated in the process of RHD-induced atrial fibrosis. Specifically, FAK mediated the process of atrial fibrosis in patients with RHD via the AKT/S6K pathway. Research on 
RHD patients and animal models uncovered that the expression of AKT/S6K signalling pathway-related factors was increased in patients with AF. Animal experiments revealed suppressed $\alpha$-smooth muscle actin ( $\alpha$-SMA) expression in TGF- $\beta 1$-induced fibroblasts after inhibition of the expression of FAK and AKT. This study (21) focused on RHD-induced fibrotic lesions. Inhibition of the expression of AKT/S6K signalling pathway-related factors inhibited TGF- $\beta 1$-induced cardiac fibroblast activation, which was confirmed in other studies (22). Both studies suggested that the AKT/S6K signalling pathway was closely associated with the fibrosis of heart tissue, and inhibition of its expression inhibited cardiac fibroblast activation. Therefore, the AKT/S6K signalling pathway may be an effective intervention target for the prevention or treatment of fibrotic lesions caused by RHD. The aforementioned study (21) again emphasizes the important role of TGF- $\beta 1$ in fibrotic lesions due to RHD.

TGF- $\beta 1 /$ Smad signalling pathway and RHD. Zhang et al (23) also found that the TGF- $\beta 1 /$ Smad signalling pathway increased the expression of $\alpha$-actinin- 2 and participated in the process of RHD-induced atrial fibrosis. Patients with RHD-induced AF were used as the experimental group and patients with congenital heart disease and SR who underwent heart surgery were used as controls. The atrial specimens of the two groups were compared. The levels of TGF- $\beta 1 /$ Smad signalling pathway-related factors and $\alpha$-actinin- 2 in the experimental group were significantly higher compared with those in the control group. The TGF- $\beta 1 / \mathrm{Smad}$ signalling pathway is closely associated with scar tissue formation, and disruption of the TGF- $\beta 1 /$ Smad pathway is an important pathogenic mechanism of tissue fibrosis (24). The results of a previous study (23) suggest that disorder of this pathway caused atrial fibrosis. However, the exact cause of TGF- $\beta 1 /$ Smad pathway dysfunction is not known, and the upstream stimuli for this pathway must be investigated in future studies. This study also highlights the important role of TGF- $\beta 1$ in RHD-induced fibrosis. TGF- $\beta 1$ was the focus of a previous study (19) on the association between the MAPK signalling pathway and RHD and the association between the AKT/S6K signalling pathway and RHD. In the aforementioned study by Zhang et al (23), it was once again shown that TGF- $\beta 1$ may play an important role in the pathogenesis of RHD. The process of fibrotic valve injury during the pathogenesis of RHD is also regulated by the general fibrosis status of the body, and it is not an independent process. The view of this study (23) is that the increased expression of Smad2 and Smad3, which causes atrial fibrosis, is due to the regulation of TGF- $\beta 1$. Smad 2 and Smad 3 are also key factors in endothelial-to-mesenchymal transition (EMT), as the phosphorylation of Smad2 and Smad3 regulates the transcription factors lymphoid enhancer factor-1, Snaill, TWIST and zinc finger E-box binding homeobox $1 / 2$ to induce EMT $(25,26)$. However, although EMT was not the focus of this study (23), it suggests that the occurrence and development of RHD may involve EMT.

Wnt signalling pathway and RHD. Guo et al (27) found that the Wnt signalling pathway increased the expression of Snaill and participated in the process of RHD-induced atrial fibrosis. The method they used was similar to the three studies on
MAPK mentioned above, i.e., comparison of the SR and AF groups of patients with RHD. The Wnt signalling pathway involves multiple signal molecule-mediated transduction pathways, and the activation of this pathway is observed in several cardiovascular pathologies (28). This study (27) extended the prevalence of the Wnt signalling pathway in cardiovascular disease to RHD and highlighted Snaill. The Wnt signalling pathway and Snaill are implicated in the EMT process. The Wnt signalling pathway is closely associated with EMT. For example, the activation of the Wnt signalling pathway after myocardial infarction triggers EMT to promote the cardiac repair process (29). Snaill is one of the transcription factors that induces EMT $(25,26)$. These data suggest that EMT may play a role in the pathogenesis of RHD. Therefore, whether the results of this research (increased expression of Snaill participated in the process of RHD-induced atrial fibrosis) include the participation of EMT requires future in-depth investigation.

Signal transducer and activator of transcription 3 (STAT3) signalling pathway and RHD. Wu et al (3) demonstrated that the sphingosine 1-phosphate receptor 1 (S1PR1)/STAT3 signalling pathway was activated during RHD-induced valve damage. The authors established an RHD rat model to detect differences in the expression of S1PR1, STAT3, phosphorylated (p)-STAT3 and T helper (Th) 17 cell-related cytokines in the cardiac valves of RHD and control rats, and found that the expression of S1PR1 in the cardiac valves of RHD rats was lower compared with that in controls. The expression levels of p-STAT3 and the Th17 cell-related cytokines interleukin (IL)-17 and IL-21 were higher compared with those in the control group. The inflammation and fibrosis of the valves in the RHD group were also more prominent compared with the control group.

Chen et al (30) found that inhibiting the expression of miR-155-5p inhibited the expression of p-STAT3 in an RHD rat model and reduced the expression of the Th17 cell-related cytokines IL-6 and IL17 and valve fibrotic damage and inflammation caused by RHD.

The focus of both studies was on STAT3-related signalling pathways and valvular disease due to RHD. Valvular disease is the hallmark of RHD, which may also cause other secondary heart diseases. These two studies directly investigated valvular disease, which is relatively more meaningful in the pathogenesis of RHD. The research content of these two studies gradually deepened the understanding of STAT3 signalling pathway. The first study observed that the STAT3 signalling pathway was activated in the RHD rat model, and the second study examined methods for interfering with the STAT3 signalling pathway.

STAT3 is a cellular signal transcription factor that is involved in numerous cell activities, and has been extensively investigated (31). Both studies emphasized that the main role of STAT3 in RHD-induced valve damage was to induce CD4 ${ }^{+}$ T-cell differentiation into Th17 cells, which was described in previous studies (32). The important role of $\mathrm{CD} 4^{+} \mathrm{T}$ cells in the pathogenesis of RHD is therefore confirmed.

Both studies investigated Th17 immune cells and their specific mechanism of action, which included the increased phosphorylation of STAT3 to promote the differentiation of 


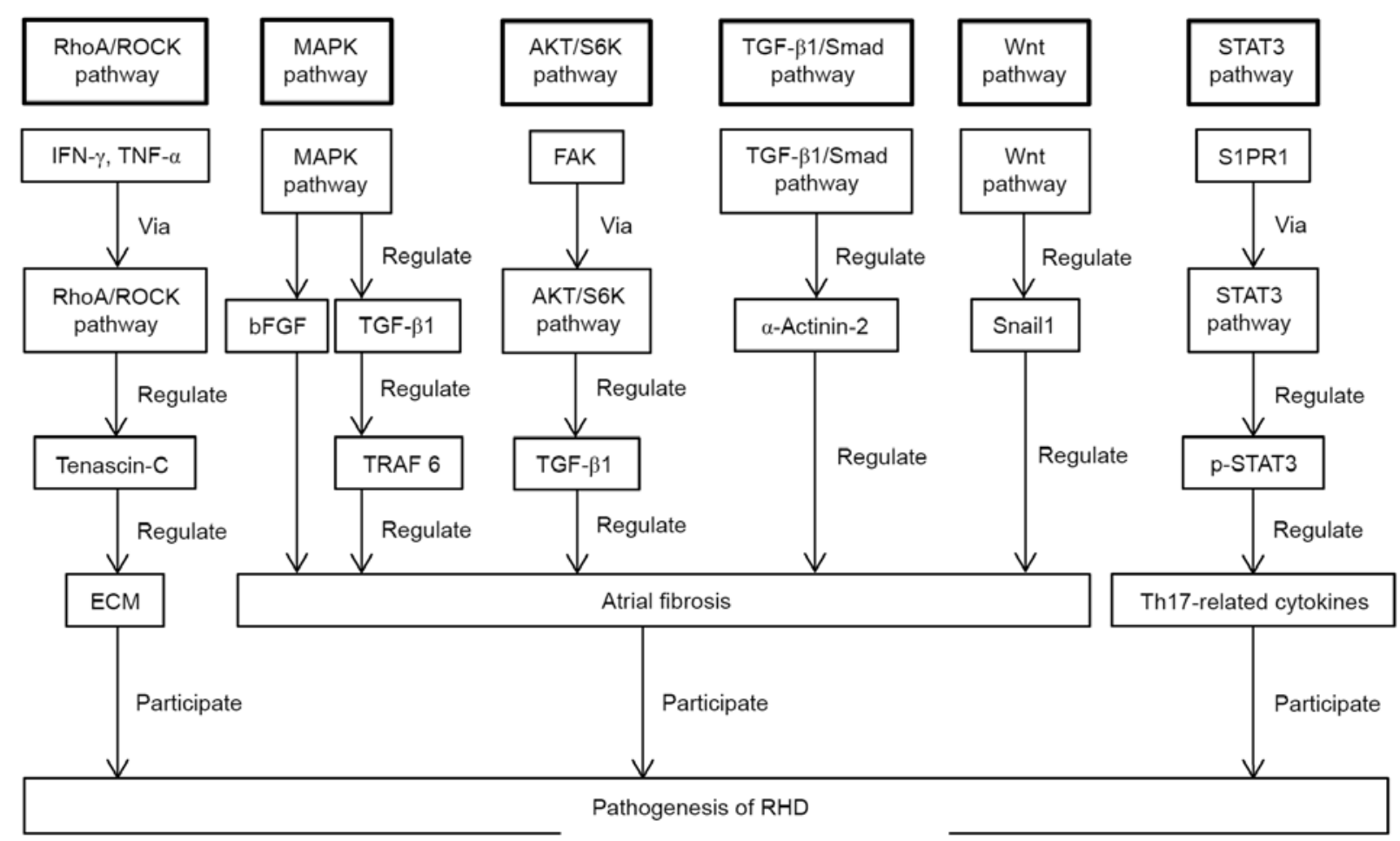

Figure 1. Schematic presentation of the discussed signalling pathways that are implicated in the pathogenesis of RHD. RHD, rheumatic heart disease; RhoA/ROCK, RhoA/Rho-dependent kinase; IFN- $\gamma$, interferon- $\gamma$; TNF- $\alpha$, tumour necrosis factor- $\alpha$; ECM, extracellular matrix; MAPK, mitogen-activated protein kinase; bFGF, basic fibroblast growth factor; TGF- $\beta 1$, transforming growth factor- $\beta 1$; TRAF 6 , tumour necrosis factor receptor-associated factor 6 ; AKT/S6K, protein kinase B/S6 kinase; FAK, focal adhesion kinase; S1PR1, sphingosine 1-phosphate receptor 1; STAT3, signal transducer and activator of transcription 3; p-STAT3, phosphorylated STAT3; Th17, T helper 17.

$\mathrm{CD} 4^{+} \mathrm{T}$ cells into Th17 cells, which release the inflammatory factors that participate in valve damage induced by RHD. Both studies involved animal experiments, but an increase in the Th17 cell-related cytokine IL-17A was also reported in RHD patients (33). Therefore, Th17 cells play an important role in RHD-induced valve inflammation.

In summary, the two studies $(3,30)$ focused on the important role of the STAT3 signalling pathway and Th17 cells in the process of valve inflammation and fibrosis caused by RHD. The mechanism of valve injury in RHD is closely associated with inflammatory factors, and STAT3-related signalling pathways play an important role in regulating related inflammatory factors. miR-155-5p may be an effective intervention target. Further research into the STAT3 pathway may uncover that this pathway may represent a promising target for the prevention or treatment of RHD with.

\section{Discussion and conclusions}

The conclusions of the studies on these signalling pathways associated with the pathogenesis of RHD may be summarized as follows:

Multiple signalling pathways regulate the pathogenesis of RHD. A schematic diagram of the role of the signalling pathways described in this review in the pathogenesis of RHD is presented in Fig. 1.

These signalling pathways serve their respective physiological or pathological roles in the occurrence and development of RHD. Some of these signalling pathways play the same role in the occurrence and development of RHD as in other diseases.
However, further research is needed to determine whether the functions of these signalling pathways in other diseases are also involved in the occurrence and development of RHD. In the future, the present review may help determine the impact of new RHD prevention and treatment methods on other diseases that use the same signalling pathways.

The pathogenesis of RHD may not be a simple immune response but may involve other conditions, including dysfunction of signalling pathways, such as the TGF- $\beta 1 / \mathrm{Smad}$ signalling pathway. Targeted intervention in the expression process of some of the signalling pathways may reduce the heart damage caused by RHD. The pathogenesis of RHD involves several pathological processes, primarily inflammatory reactions and fibrosis. Multiple signalling pathways regulate each process, such as atrial fibrosis. $\mathrm{CD} 4^{+} \mathrm{T}$ cells are the main immune cells in the pathogenesis of RHD. Important factors in the occurrence and development of RHD include bFGF, TGF- $\beta 1$ and $\mathrm{CD} 4^{+} \mathrm{T}$ cells.

EMT is most likely implicated in the occurrence and development of RHD and its role requires further investigation. Some progress has been made in the field of RHD-related signalling pathway research. Unfortunately, only research on the RhoA/ROCK, AKT/S6K and STAT3 signalling pathways identified intervention targets, and the effect of such intervention in humans requires further investigation.

There are currently three intervention targets that may be used for the prevention or treatment of RHD: Intervention in IFN- $\gamma$ and TNF- $\alpha$-mediated ECM remodelling; intervention in the AKT/S6K pathway to suppress $\alpha$-SMA expression in TGF- $\beta 1$-induced fibroblasts; and interference with the 
phosphorylation of STAT3 to inhibit Th17 cell-related cytokine release and reduce RHD-induced valve damage.

Each RHD-related signalling pathway study described in the present review has limitations. No specific mechanism for the regulation of each signalling pathway on RHD was investigated in depth. If the specific mechanisms of the regulation of each signalling pathway in RHD are further investigated on the basis of existing research and further effective drug intervention targets are identified in humans, the prevention and treatment of RHD may improve.

There is some cross-talk between these signalling pathways. The MAPK, AKT/S6K, TGF- $\beta 1 /$ Smad and Wnt signalling pathways are all involved in the pathogenesis of RHD via regulation of atrial fibrosis; both the MAPK and AKT/S6K signalling pathways regulate TGF- $\beta 1$; both the MAPK and STAT3 signalling pathways regulate $\mathrm{CD} 4^{+} \mathrm{T}$ cells; the TGF- $\beta 1 / \mathrm{Smad}$ and Wnt signalling pathways have the potential to regulate EMT in the pathogenesis of RHD.

There was a limitation to the present study. Meta-analysis has statistical power and a better ability to extrapolate to the greater population, and it should be performed in our future studies further elucidate the pathogenesis of RHD.

The number of studies on the pathogenesis of RHD worldwide is limited. Although the researchers mentioned in this article did not further examine the mechanism of action of these signalling pathways based on existing research, their efforts have produced valuable results, which will prompt future research that may ultimately fully elucidate the pathogenesis of RHD and identify prevention and treatment methods accessible to patients in underdeveloped areas.

\section{Acknowledgements}

Not applicable.

\section{Funding}

The present study was supported by the National Natural Science Foundation of China (grant no. 81960082), the Guangxi Key Laboratory Base of Precision Medicine in Cardio-cerebrovascular Disease Control and Prevention (grant no. 17-259-85), the Guangxi Clinical Research Center for Cardio-cerebrovascular Diseases (grant no. AD17129014) and the Guangxi Medical High-level Backbone Talents '139' Program (grant no. G201901006).

\section{Availability of data and materials}

All data generated or analyzed during the present study are included in this published article.

\section{Authors' contributions}

$\mathrm{ZZ}$ and SX conceived and designed the study. SX collected and analyzed the data. SX wrote the manuscript. All the authors have read and approved the final manuscript.

\section{Ethics approval and consent to participate}

Not applicable.

\section{Patient consent for publication}

Not applicable.

\section{Competing interests}

The authors declare that they have no competing interests.

\section{References}

1. Leal MTBC, Passos LSA, Guarçoni FV, de Souza Aguiar JM, da Silva RBR, de Paula TMN, Santos RFD, Nassif MCL, Gomes NFA, Tan TC and Nunes MCP: Rheumatic heart disease in the modern era: Recent developments and current challenges. Rev Soc Bras Med Trop 52: e20180041, 2019.

2. Mirabel M, Narayanan K, Jouven X and Marijon E: Cardiology patient page. Prevention of acute rheumatic fever and rheumatic heart disease. Circulation 130: e35-e37, 2014.

3. Wu XD, Zeng ZY, Gong DP, Wen JL and Huang F: Potential involvement of S1PR1/STAT3 signaling pathway in cardiac valve damage due to rheumatic heart disease. Biotech Histochem 94: 398-403, 2019.

4. Wang Y, He G, Tang H, Shi Y, Kang X, Lyu J, Zhu M, Zhou M, Yang M, Mu M, et al: Aspirin inhibits inflammation and scar formation in the injury tendon healing through regulating JNK/STAT-3 signalling pathway. Cell Prolif 52: e12650, 2019.

5. Liu CH, Hua N, Fu X, Pan YL, Li B and Li XD: Metformin regulates atrial SK2 and SK3 expression through inhibiting the PKC/ERK signaling pathway in type 2 diabetic rats. BMC Cardiovasc Disord 18: 236, 2018.

6. Sung JY and Choi HC: Nifedipine inhibits vascular smooth muscle cell proliferation and reactive oxygen species production through AMP-activated protein kinase signaling pathway. Vascul Pharmacol 56: 1-8, 2012.

7. Jiang L, Wei XF, Yi DH, Xu P, Liu H, Chang Q, Yang SM, Li ZF, Gao HB and Hao GJ: Synergistic effects of cyclic strain and Th1-like cytokines on tenascin-C production by rheumatic aortic valve interstitial cells. Clin Exp Immunol 155: 216-223, 2009.

8. Zeisberg M, Hanai J, Sugimoto H, Mammoto T, Charytan D, Strutz F and Kalluri R: BMP-7 counteracts TGF-beta1-induced epithelial-to-mesenchymal transition and reverses chronic renal injury. Nat Med 9: 964-968, 2003.

9. Yang L, Tang L, Dai F, Meng G, Yin R, Xu X and Yao W: Raf-1/CK 2 and RhoA/ROCK signaling promote TNF- $\alpha$-mediated endothelial apoptosis via regulating vimentin cytoskeleton. Toxicology 389: 74-84, 2017.

10. Vicente-Steijn R, Kelder TP, Tertoolen LG, Wisse LJ, Pijnappels DA, Poelmann RE, Schalij MJ, deRuiter MC, Gittenberger-de Groot AC and Jongbloed MRM: RHOA-ROCK signalling is necessary for lateralization and differentiation of the developing sinoatrial node. Cardiovasc Res 113: 1186-1197, 2017.

11. Li L, Zhao Q and Kong W: Extracellular matrix remodeling and cardiac fibrosis. Matrix Biol 68-69: 490-506, 2018.

12. Dziki JL and Badylak SF: Extracellular matrix for myocardial repair. Adv Exp Med Biol 1098: 151-171, 2018.

13. Diamantino Soares AC, Araújo Passos LS, Sable C, Beaton A, Ribeiro VT, Gollob KJ, Dutra WO and Nunes MCP: Circulating cytokines predict severity of rheumatic heart disease. Int J Cardiol 289: 107-109, 2019.

14. Rehman S, Akhtar N, Saba N, Munir S, Ahmed W, Mohyuddin A and Khanum A: A study on the association of TNF- $\alpha(-308)$, IL-6(-174), IL-10(-1082) and IL-1Ra(VNTR) gene polymorphisms with rheumatic heart disease in Pakistani patients. Cytokine 61: 527-531, 2013.

15. Deng H, Xue YM, Zhan XZ, Liao HT, Guo HM and Wu SL: Role of tumor necrosis factor-alpha in the pathogenesis of atrial fibrillation. Chin Med J (Engl) 124: 1976-1982, 2011.

16. Guilherme L, Cury P, Demarchi LM, Coelho V, Abel L, Lopez AP, Oshiro SE, Aliotti S, Cunha-Neto E, Pomerantzeff PMA, et al: Rheumatic heart disease: Proinflammatory cytokines play a role in the progression and maintenance of valvular lesions. Am J Pathol 165: 1583-1591, 2004. 
17. Li M, Yi X, Ma L and Zhou Y: Hepatocyte growth factor and basic fibroblast growth factor regulate atrial fibrosis in patients with atrial fibrillation and rheumatic heart disease via the mitogen-activated protein kinase signaling pathway. Exp Ther Med 6: 1121-1126, 2013.

18. Zhang D, Liu X, Chen X, Gu J, Li F, Zhang W and Zheng Y: Role of the MAPKs/TGF- $\beta 1 / T R A F 6$ signaling pathway in atrial fibrosis of patients with chronic atrial fibrillation and rheumatic mitral valve disease. Cardiology 129: 216-223, 2014.

19. Zhao Z, He D, Ling F, Chu T, Huang D, Wu H and Ge J: CD4 ${ }^{+} \mathrm{T}$ cells and TGF $\beta 1 /$ MAPK signal pathway involved in the valvular hyperblastosis and fibrosis in patients with rheumatic heart disease. Exp Mol Pathol 114: 104402, 2020.

20. Lee S, Rauch J and Kolch W: Targeting MAPK signaling in cancer: Mechanisms of drug resistance and sensitivity. Int J Mol Sci 21: 1102, 2020.

21. Zhang $\mathrm{P}$, Wang W, Wang $\mathrm{X}$, Wang $\mathrm{X}$, Song $\mathrm{Y}$, Zhang $\mathrm{J}$ and Zhao $\mathrm{H}$ : Focal adhesion kinase mediates atrial fibrosis via the AKT/S6K signaling pathway in chronic atrial fibrillation patients with rheumatic mitral valve disease. Int J Cardiol 168: 3200-3207, 2013.

22. Narikawa M, Umemura M, Tanaka R, Fujita T, Yokoyama U, Ishigami T, Kimura K, Tamura K and Ishikawa Y: Acute hyperthermia inhibits TGF- $\beta 1$-induced cardiac fibroblast activation via suppression of Akt signaling. Sci Rep 8: 6277, 2018.

23. Zhang L, Zhang N, Tang X, Liu F, Luo S and Xiao H: Increased $\alpha$-actinin-2 expression in the atrial myocardium of patients with atrial fibrillation related to rheumatic heart disease. Cardiology 135: 151-159, 2016.

24. Hu HH, Chen DQ, Wang YN, Feng YL, Cao G, Vaziri ND and Zhao YY: New insights into TGF- $\beta /$ Smad signaling in tissue fibrosis. Chem Biol Interact 292: 76-83, 2018.

25. Vincent T, Neve EPA, Johnson JR, Kukalev A, Rojo F, Albanell J, Pietras K, Virtanen I, Philipson L, Leopold PL, et al: A SNAIL1-SMAD3/4 transcriptional repressor complex promotes TGF-beta mediated epithelial-mesenchymal transition. Nat Cell Biol 11: 943-950, 2009.
26. Thuault S, Tan EJ, Peinado $\mathrm{H}$, Cano A, Heldin $\mathrm{CH}$ and Moustakas A: HMGA2 and Smads co-regulate SNAIL1 expression during induction of epithelial-to-mesenchymal transition. J Biol Chem 283: 33437-33446, 2008.

27. Guo F, Yi X, Li M, Fu J and Li S: Snaill is positively correlated with atrial fibrosis in patients with atrial fibrillation and rheumatic heart disease. Exp Ther Med 14: 4231-4237, 2017.

28. Foulquier S, Daskalopoulos EP, Lluri G, Hermans KCM, Deb A and Blankesteijn WM: WNT signaling in cardiac and vascular disease. Pharmacol Rev 70: 68-141, 2018.

29. Aisagbonhi O, Rai M, Ryzhov S, Atria N, Feoktistov I and Hatzopoulos AK: Experimental myocardial infarction triggers canonical Wnt signaling and endothelial-to-mesenchymal transition. Dis Model Mech 4: 469-483, 2011.

30. Chen A, Wen J, Lu C, Lin B, Xian S, Huang F, Wu Y and Zeng Z: Inhibition of miR-155-5p attenuates the valvular damage induced by rheumatic heart disease. Int J Mol Med 45: 429-440, 2020.

31. Hu YS, Han X and Liu XH: STAT3: A potential drug target for tumor and inflammation. Curr Top Med Chem 19: 1305-1317, 2019.

32. Liu X, Hu H, Fan H, Zuo D, Shou Z, Liao Y, Nan Z and Tang Q: The role of STAT3 and AhR in the differentiation of CD4 ${ }^{+} \mathrm{T}$ cells into Th17 and Treg cells. Medicine (Baltimore) 96: e6615, 2017.

33. Bas HD, Baser K, Yavuz E, Bolayir HA, Yaman B, Unlu S, Cengel A, Bagriacik EU and Yalcin R: A shift in the balance of regulatory $\mathrm{T}$ and $\mathrm{T}$ helper 17 cells in rheumatic heart disease. J Investig Med 62: 78-83, 2014.

This work is licensed under a Creative Commons Attribution-NonCommercial-NoDerivatives 4.0 International (CC BY-NC-ND 4.0) License. 